\title{
UNRAVELING KEY INTERACTIONS THAT LEADS THE STABILITY OF NBD-14010 BINDING TO GP120 OF HIV-1: AN MD SIMULATIONS AND FREE ENERGY PERSPECTIVES
}

\author{
Vishnudatt Pandey ${ }^{1, *}$, Gargi Tiwari ${ }^{1}$, Rakesh K. Tiwari ${ }^{1}$ \\ and Rajendra Prasad Ojha ${ }^{1^{*}}$ \\ ${ }^{1}$ Biophysics Unit, Department of Physics, Deen Dayal Upadhyay Gorakhpur University, \\ Gorakhpur, Uttar Pradesh, India, 273009 \\ *E-mail: vishnudattpandey127@gmail.com
}

\begin{abstract}
The contact between gp120 and CD4 binding site leads the HIV-1 entry into the host membrane and therefore, gp120 and CD4 binding sites become a fertile target for many drug discovery projects. The drug NBD-14010, like its predecessor NBD-11021 is a small molecule that can stuff the interactions between gp120 and CD4, and has shown good clinical potency. MD simulation and free energy calculations are used in the present study to focus the thermodynamical insight of binding of NBD-14010 in CD4 binding sites. The energetic of binding of NBD-14010 clearly shows a hydrophobic interaction driven binding of the drug which specifically targets CD4 binding loops. The residue wise free energy decomposition reveals Asn 286 and Trp 288 as key residues stabilizing the binding of NBD-14010 while indicating few more important amino acids in the vicinity of the Phe 43 cavity which may also be targeted for further inhibition of HIV-1 entry.
\end{abstract}

Keywords: 82M; Free Energy Calculations; HIV-1 entry; gp120 binding; MD simulations

() RASĀYAN. All rights reserved

\section{INTRODUCTION}

Acquired immune deficiency syndrome, which is because of a retro-virus termed as human immunodeficiency virus (HIV), is a serious health issue for mankind ${ }^{1,2}$, and so it becomes a fertile target for medicinal chemists. Since the entry of HIV is mediated by conformational changes in an outermost subunit of virion i.e. envelope protein, it becomes a primary goal for conformational and structural studies. The structural studies reveal HIV type 1 (HIV-1) envelope protein as an assembly of two glycoproteins: gp120 and trans-membrane glycoprotein gp $41^{3,4}$. Binding of gp120 to the receptor protein, $\mathrm{CD} 4$, is initiated and gives rise to the exposure of its potency towards the binding-site to the co-receptor CCR5 or CXCR4 ${ }^{5,6}$. The In-silico simulation of gp120 and its binding with receptor CD4 and co-receptor plays an important role in virus infection and immune system ${ }^{7}$. Its entry process includes a number of conformational patterns of gp120 such as its interactions with receptor and co-receptor. The envelope glycoproteins gp120 and gp41 were obtained from a common precursor, gp160, which breaks during transport into two main components ${ }^{3}$. A trimetric complex was formed through non-covalent interaction between three gp120 and three gp41 molecules known as a viral spike. As the curiosity about the mechanism of virus entry progressed, there are several structures have been reported by crystallographic studies $^{8,12}$. In the same reference, Curreli and coworkers found NBD-14010 and its derivatives as the best compounds ${ }^{7}$ to test against a series of 51 Env-pseudotyped HIV- ${ }^{13}$. These compounds are inhibited cellto-cell fusion and cell-to-cell HIV-1 transmission. This study is expected to good step for new ways of designing more strong and effective HIV-1 entry inhibitors. As suggested from the x-ray structure of NBD-11021, it bounds to HIV-1 env gp120 and gives rise to a conclusion that this molecule binds to the Phe43 cavity ${ }^{14}$. The energetic contribution of each amino acid was verified at the binding site involved in stabilizing the complex, ( PDB: 5U6E) $)^{15}$ some important amino acids are associated with stable proteinligand complexes, and are involved in the interaction with inhibitors which could be very important for 
structure-based drug discovery. Although, they do not provide deeper insights for each residue. Thus, it is necessary to disclose crucial properties of some important amino acids which play the main role in stabilizing protein-ligand complexes. These studies may lead us to a very important conclusion of novel drug discoveries. Decomposition energy, Molecular dynamics (MD) simulations and Molecular Mechanics Generalized Born Surface Area (MMGBSA) binding free energy calculations and are some powerful computer-based tools to provide deep investigations and to exploit the virus mechanism.

\section{EXPERIMENTAL}

The available crystal structure of a gp120 protein with ligand $82 \mathrm{M}$ will be taken from protein data bank (PDB id 5U6E) $)^{15}$. The parameters and missing hydrogen for gp120 were prepared using LEAP module of AMBER package ${ }^{16}$ using amber ff12SB force filed. Since the parameter for the ligand $82 \mathrm{M}$ was not available, the Antechamber module of the Amber 12 was used for the preparation of parameters. Geometry optimization of $82 \mathrm{M}$ was performed by the Gaussian 09 package using Hartree Fock (HF)/6$31 \mathrm{G}^{*}$ basis set. Restrained electrostatic potential method (RESP) ${ }^{17,18}$ for the calculation of partial atomic charges was used. With the help of $\mathrm{Na}+$, the system was neutralized, thereafter kept in TIP3P water octahedral box surrounding the protein and extending $10 \AA$ from its exterior. Then the system was annealed from 10 to $300 \mathrm{~K}$ over a period of $200 \mathrm{ps}$ and then a target temperature of $300 \mathrm{~K}$ was maintained in the isothermal-isobaric ensemble (NPT), and target pressure of 1 bar using Langevin thermostat ${ }^{20}$ and Barendsen barostat ${ }^{21}$ with a collision frequency of $2 \mathrm{ps}$ and pressure relaxation time as $1 \mathrm{ps}$. The hydrogen bonds were constrained using SHAKE ${ }^{22}$. Particle mesh Ewald (PME) ${ }^{23}$ method was used to treat longrange electrostatic interaction. When the system got a temperature and pressure of $300 \mathrm{~K}$ and $1 \mathrm{bar}$, the dynamics was continued up to $4 \mathrm{~ns}$ for equilibration dynamics, with the help of discussed parameters. Thereafter, molecular mechanical production phase was initiated and continued for another $100 \mathrm{~ns}$ for the protein-ligand system. The structures in the trajectories were collected at every 10ps intervals. All analysis of trajectories were done with the Ptraj module of Amber12 while VMD 1.6. $7^{24}$, and Chimera$1.5^{25}$ graphical programs were used for the visualization.

\section{Free Energy Calculations}

$\mathrm{MM}-\mathrm{PB} / \mathrm{SA}$ approach is used for the calculations of the thermodynamic portion and free energy of binding. All the principles of MM-PB/SA methods are well established and have been discussed elsewhere ${ }^{26,27,28}$. We are using this method because it has been successfully applied to a similar system as in present study but of different class in the previous studies ${ }^{29,30,31,32}$. The composition of free energy used for binding can be understood by following terms:

Here, $\Delta \mathrm{G}_{\text {ele }}$ : electrostatic,

$$
\Delta \mathrm{G}_{\mathrm{total}}=\Delta \mathrm{G}_{\mathrm{ele}}+\Delta \mathrm{G}_{\mathrm{vdw}}+\Delta \mathrm{G}_{\mathrm{pol}}+\Delta \mathrm{G}_{\mathrm{nonpol}}
$$

$\Delta \mathrm{G}_{\mathrm{vdw}}$ : van der Waals components of the gas phase molecular mechanics free energy difference

$\Delta \mathrm{G}_{\mathrm{pol}}$ : electrostatic polar components of the salvation free energy, and

$\Delta \mathrm{G}_{\text {nonpol }}$ : a non-polar component of the salvation free energy.

Amber 12 is used to implement the MMPB/GBSA method in the calculation. Entropic calculations ($\mathrm{T} \Delta \mathrm{S}$ ) were performed using NMODE module of Amber 12 at temperature $300 \mathrm{~K}$. Before the application of MMGBSA analysis, sodium ions and all water molecules were deleted from the trajectory. The dielectric constants were $1 \& 80$ which was used for the solute and surrounding solvent.

\section{RESULTS AND DISCUSSION}

\section{Stability of Complexes during Molecular Dynamics Simulation, RMSD Analysis}

The root means square deviations (RMSD) may be used to validate the structural stability of complexes during entire simulations, particularly for comparative simulations of two similar complexes (ligand bound and free) simulated at same physical conditions. Therefore we have analyzed RMS deviations for backbone, residue-near-binding site and ligand before and after binding which are shown in Figure 1, to 
study the effect of ligand binding on the receptor. Looking at Fig.-1, we notice that the RMS deviations for ligand are not constant during MD simulations which may indicate less stable binding of the ligand to the protein. Protein backbone RMSD is relatively less flexible in ligand-bound complex relative to the ligand-free complex which may be inferred due to ligand binding. However the RMS deviation for the backbone atoms are quite stable in ligand- bound and free states which show a well converged MD trajectories. Interestingly, the RMS deviations of residues near the binding site are significantly high in absence of ligand while they are quite constant due to ligand binding. This clearly indicates that ligand binding significantly stabilizes the nearby residues on the binding. To further qualify the effect of ligand binding in specific subunits of gp120 (e.g. N-terminal, CD4 binding sites, V4 and V5 loops), we supplemented our results with RMS deviations of individual subunits as shown in Fig.-2. For a better understanding of topology for this subunit, we provide Fig.- S1. We can easily notice that ligand binding barely affects the stability of $\mathrm{N}$ terminal and V5 loops as they are quite distant from the ligand binding site. Interestingly, ligand binding does stabilize the CD4 binding site but the RMSD for the V4 loop with reference to initial structure significantly increases.

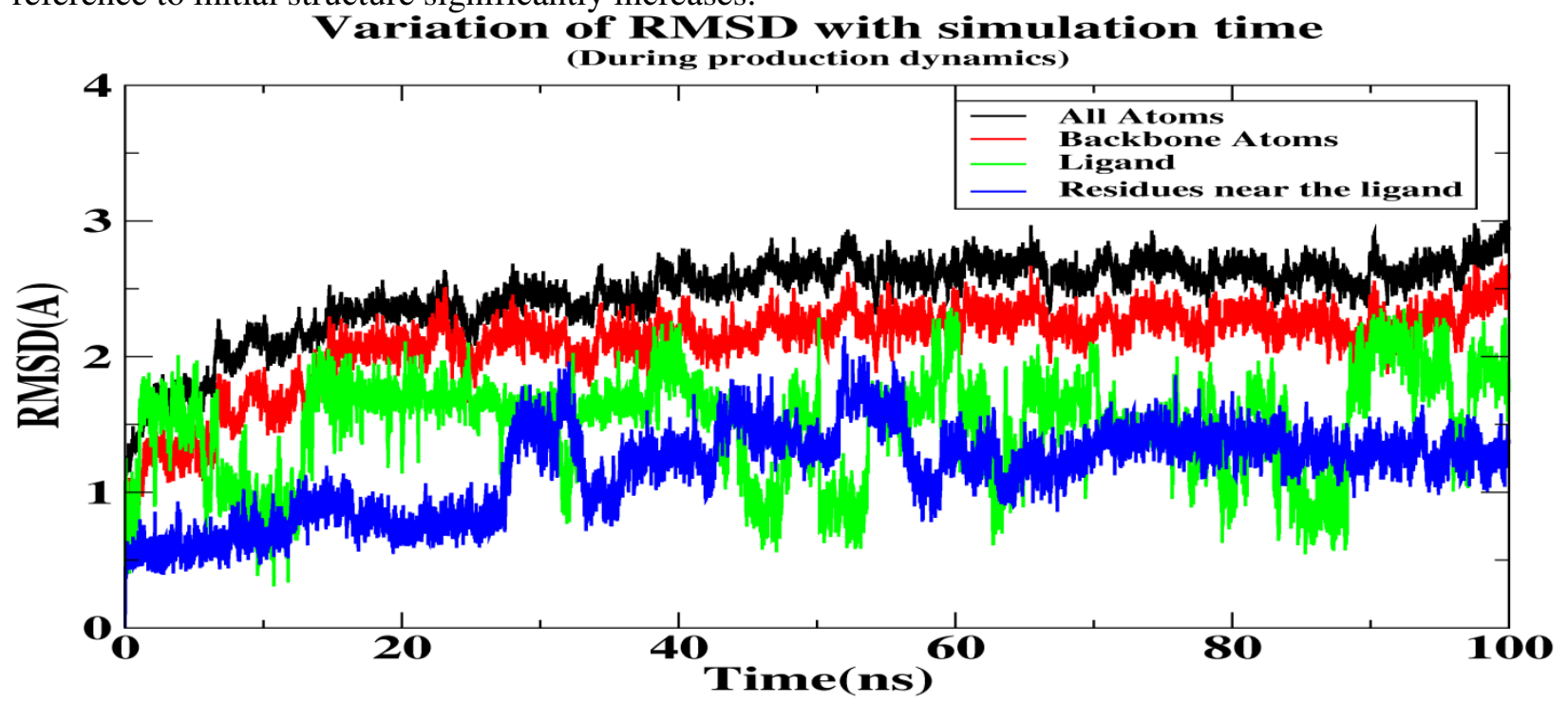

(a)

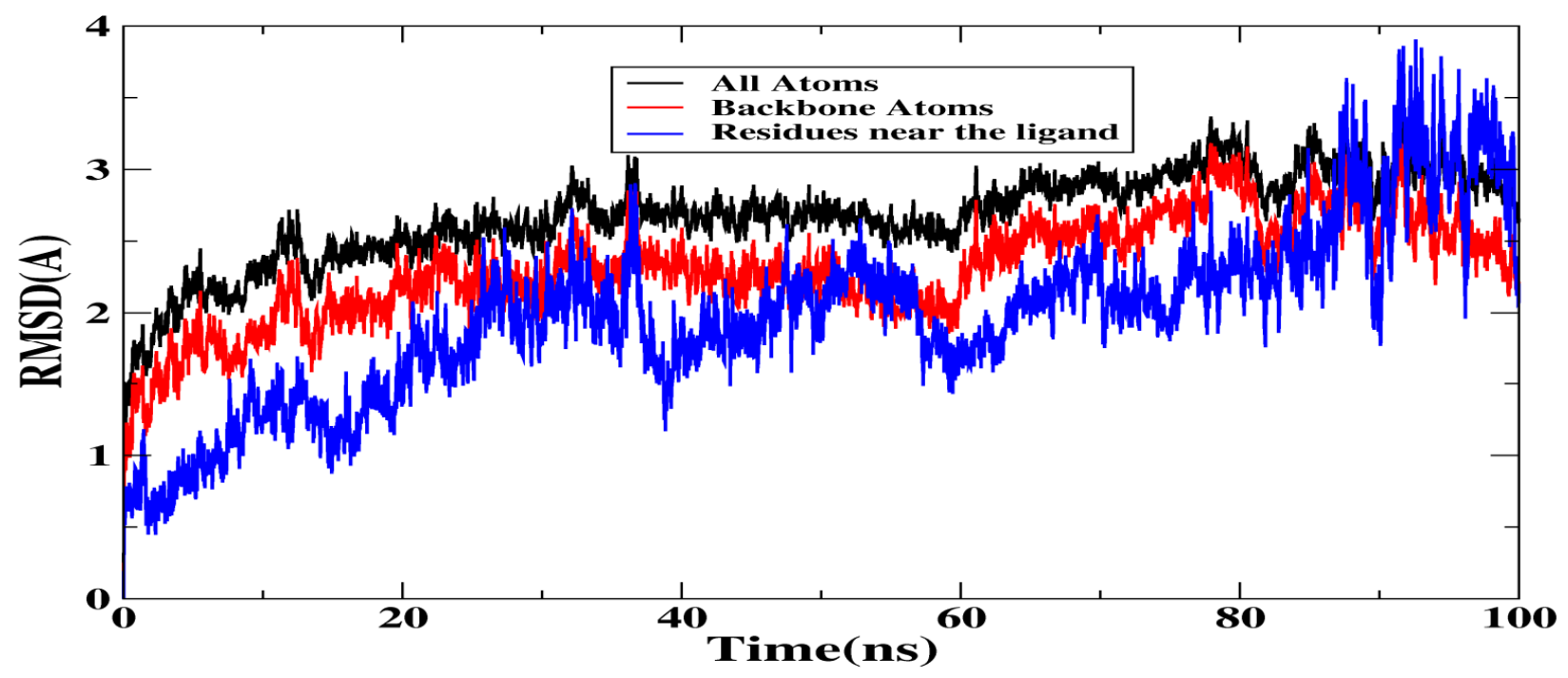

(b)

Fig.-1: (a) RMS deviation different units (named in legand) in substrate bound, (b) substrate free complex. 
The stability of CD4 site due to ligand binding is quite obvious since ligand forms many good contacts with nearby residues residing in the vicinity of the CD4 site (see Fig.-S1). We can see that $\mathrm{H} 112$ atom of ligand forms a very strong $\mathrm{H}$ bond with an OD2 atom of residue ASP235 of the CD4 site. This H bond pulls the CD4 binding site more closely to the ligand in ligand-bound conformation at a later stage of MD simulations which weakens the V4-CD4-binding loop interactions at later time of simulations, and hence we notice a significant increase in RMSD of V4 with reference (initial) structure in ligand-bound. Here we note that RMSD is a measure of the deviation from the reference structure but it doesn't report the residue fluctuations, therefore we have supplemented our study with the root-mean-square fluctuation (RMSF) of protein residues, shown in supporting Fig.-3, to account residue-wise thermal fluctuation during dynamics. RMSF shows the N-terminal and V5 loop (shown by red and thick worm) shows highest flexibility during simulations.

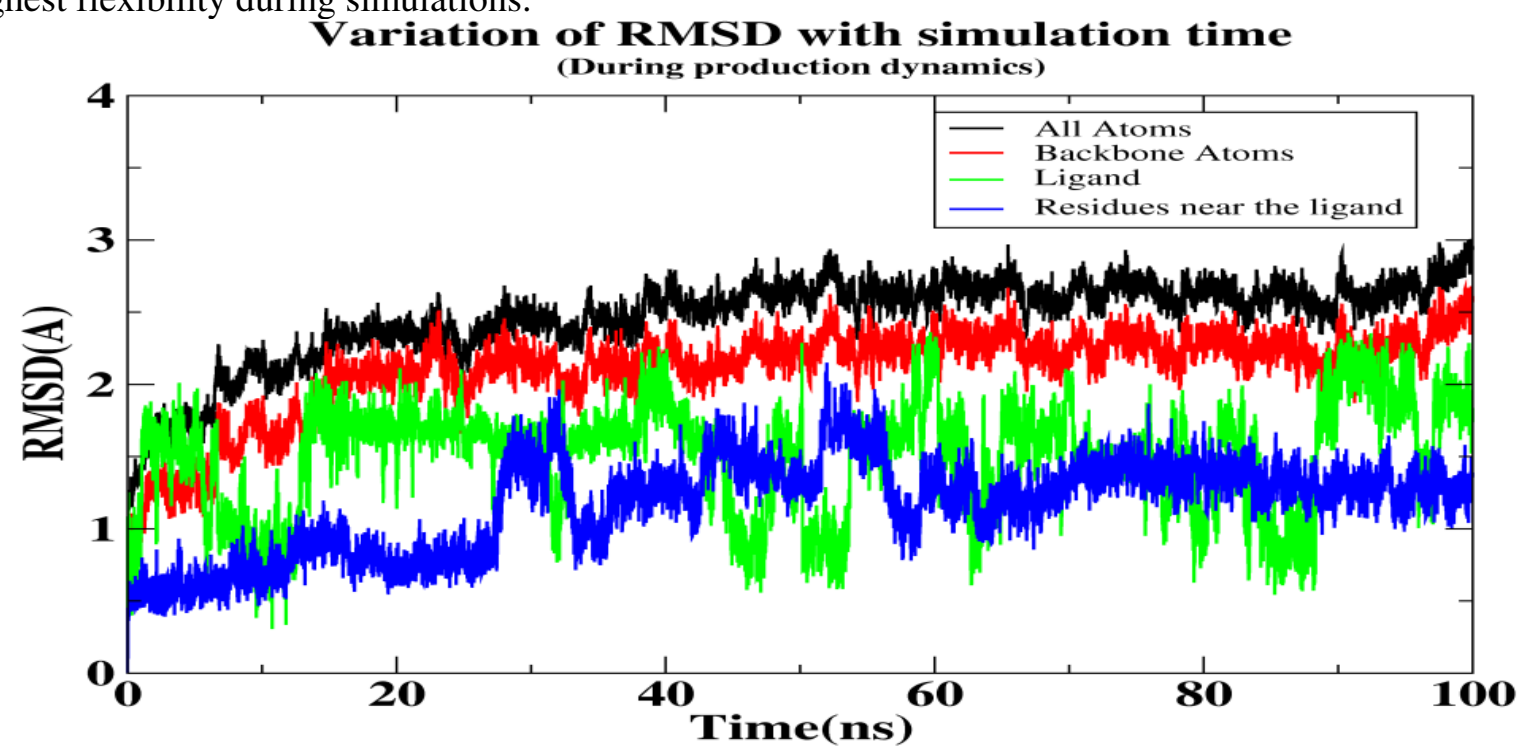

(a)

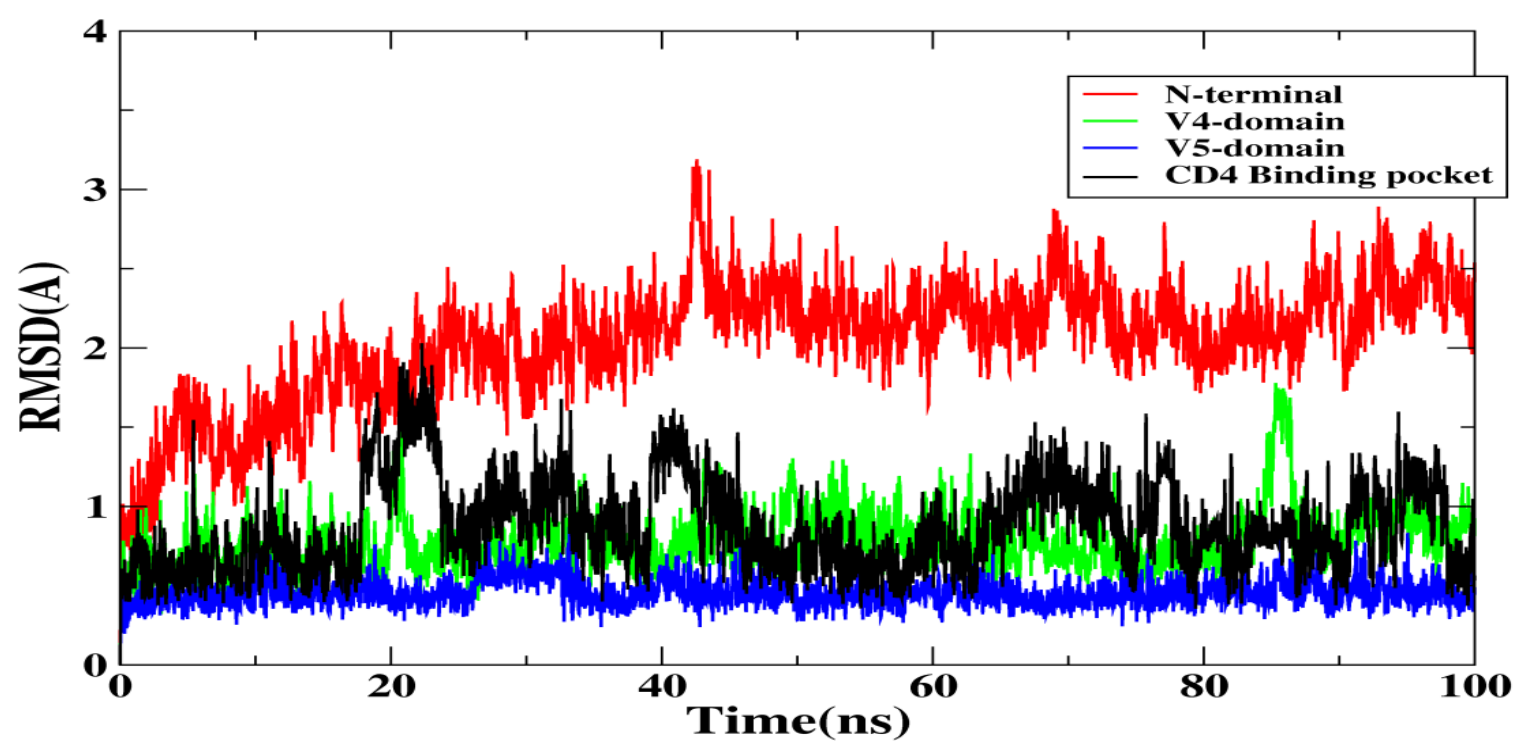

(b)

Fig.-2: RMS deviations of different subunit of protein in (a) substrate bound, (b) substrate free

We see that with the help of RMSD and RMSF we can qualify the dynamics and stability of the proteinligand complex but we do not have the quantitative insight of ligand effect on binding, therefore we have performed the thermodynamical calculations which will be discussed in next section. 
RASĀYAN J. Chem.

Vol. 11 | No. 1 | 405 - 412 | January - March | 2018

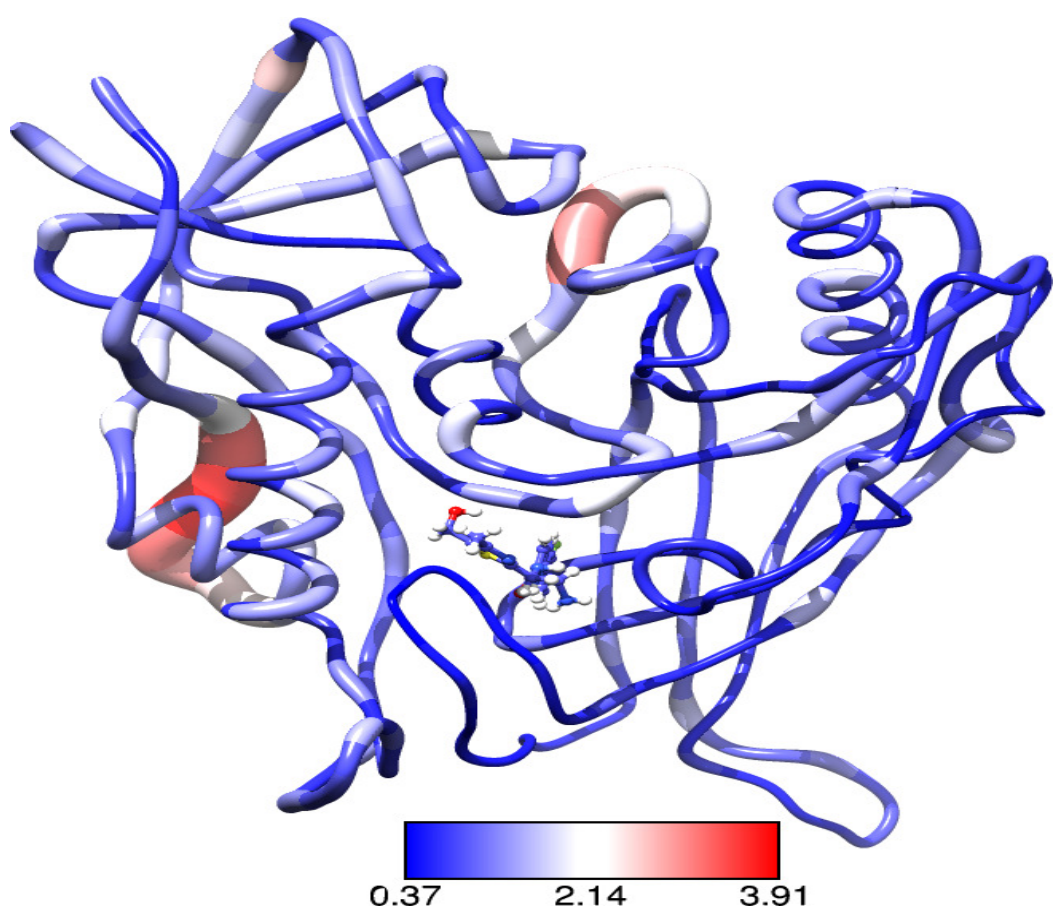

Fig.-3: Root mean square fluctuations (RMSF) of protein residues during MD simulations.

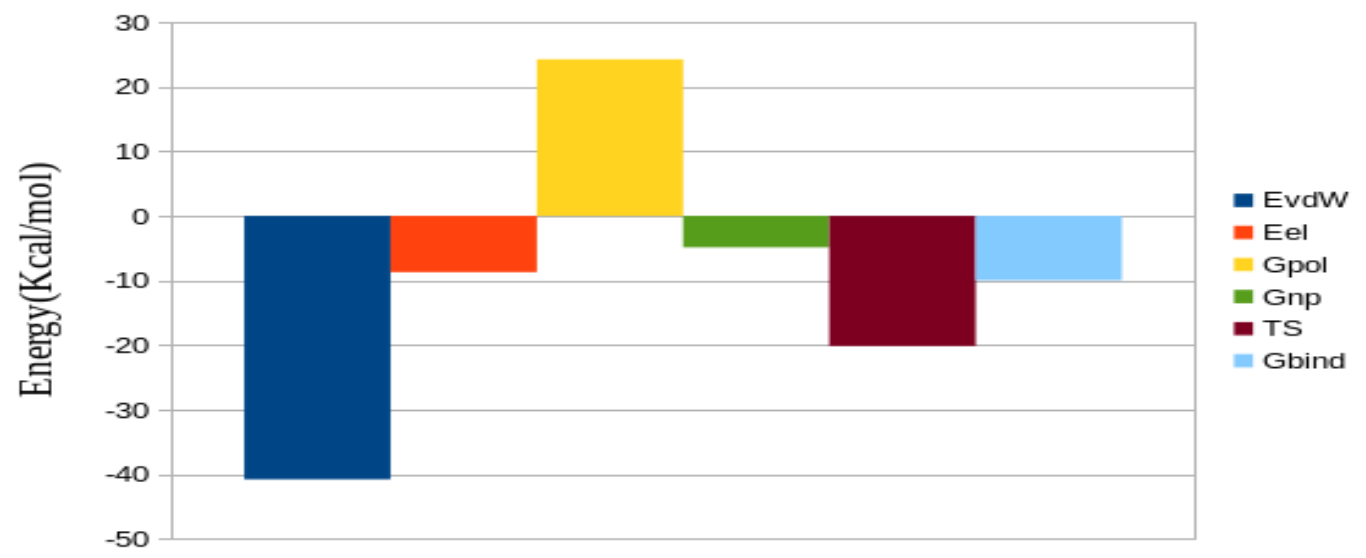

(a)

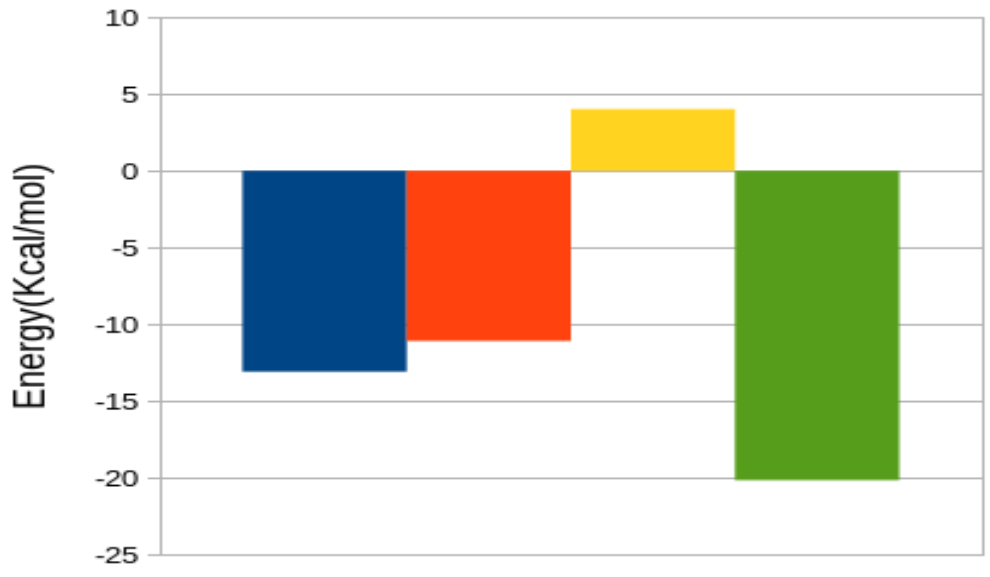

Translational

- Rotational

Vibrational

$\mathrm{T} \Delta \mathrm{S}$

(b) 


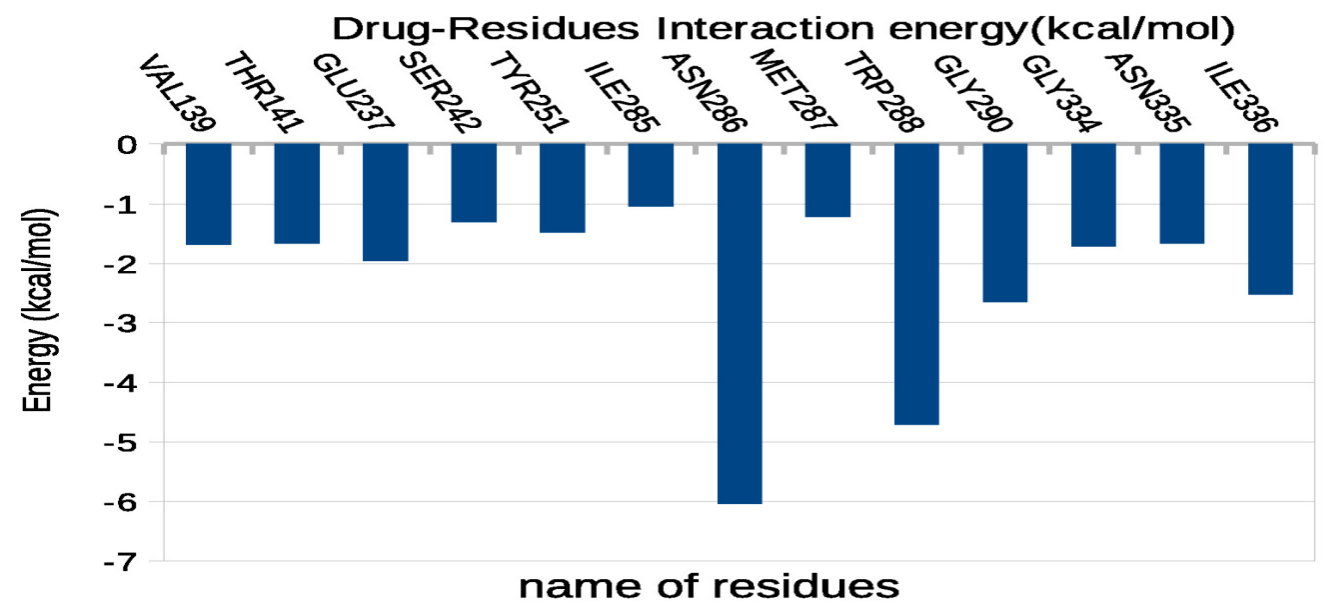

(c)

Fig.-4: (a) Energy Component for Binding of Substrate with Receptor, (b) Entropy Components for substrate

Binding calculated by MMPBSA method, (c) Residuewise energy decomposition on substrate binding.

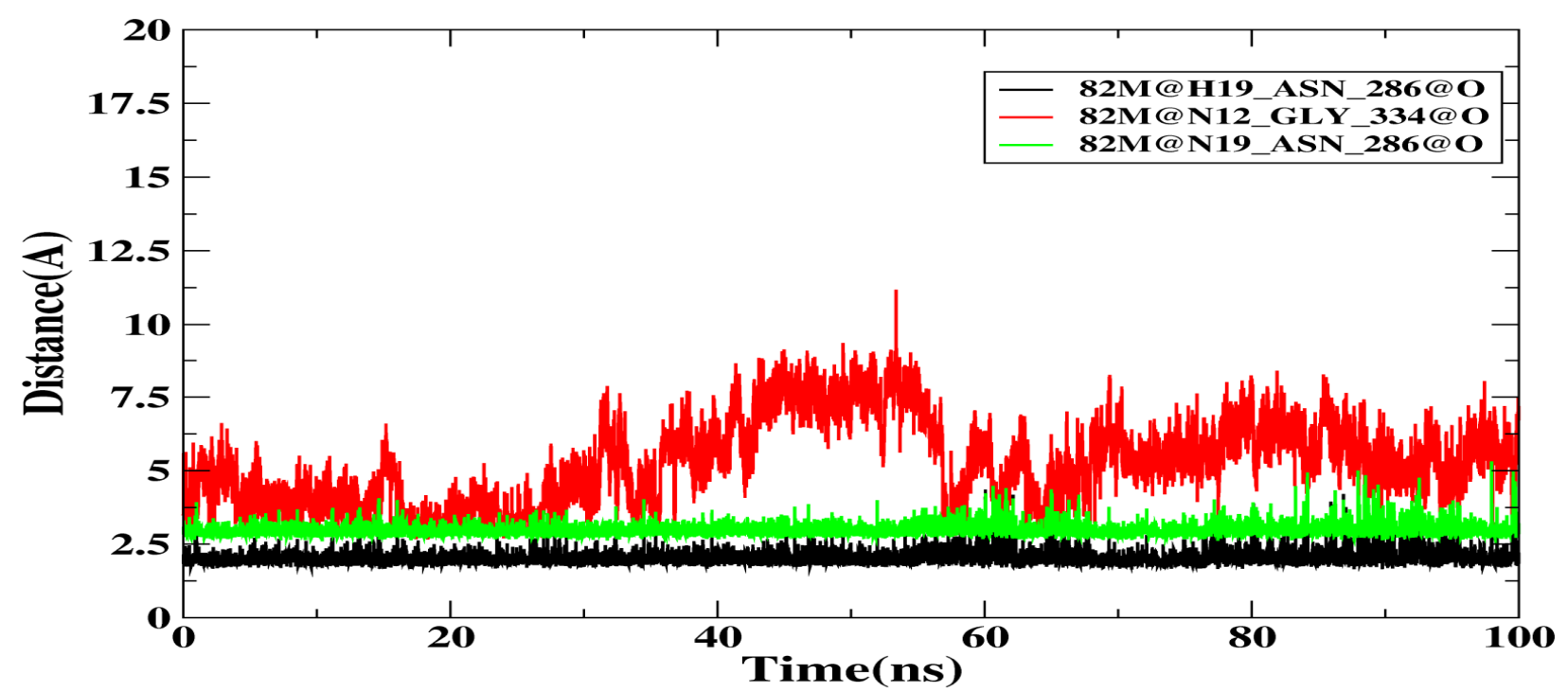

Fig.-5: Hbond distance between drug 82M and receptor during dynamics

\section{Thermodynamics of Ligand Binding}

The change in thermodynamical parameters on ligand associations was calculated by MMPBSA method, and is shown in Fig.-4a. The energy contributions shown in Fig.-4a depicts that the association of ligand with the receptor is mainly motivated by vander Waals and non-polar interactions. The electrostatic interaction is relatively weak and polar interactions are unfavorable. The total binding energy $\Delta \mathrm{G}_{\text {bind }}$ for the ligand is $-9.96 \mathrm{kcal} / \mathrm{mol}$ which shows a high binding for gp120. Since there is always an entropyenthalpy defense mechanism in ligand associations, we also calculated the entropic contributions (T $\Delta S$ ) as well, shown in Fig.-4b. This Figure shows a complex formation which is characterized by unfavorable entropy values due to a reduction in the degrees of freedom, mainly the transnational and rotational degrees of freedom. To disclose the role of each residue in the binding affinity of ligand, we calculated the residue-wise energy decomposition as shown in Fig.-4c. Figure-4c shows, it is quite obvious that Asn 286 and Trp288 have the highest contribution to the binding affinity. Backbone oxygen of Asn286 was found to form a very strong hydrogen bond with one of the amide nitrogen of the ligand $82 \mathrm{M}$. A plot showing the distance of this bond is shown in Fig.-5. Try288 is bulky residues containing aromatic side 
chain and can contribute significantly by van der Waals interaction with ligand 82M. Moreover, there are other residues like VAL139, THR141, GLU237, SER242, TYR251, ILE285, GLY290, GLY334, ASN335 and ILE336 which also interacts with protein rather weak interaction.

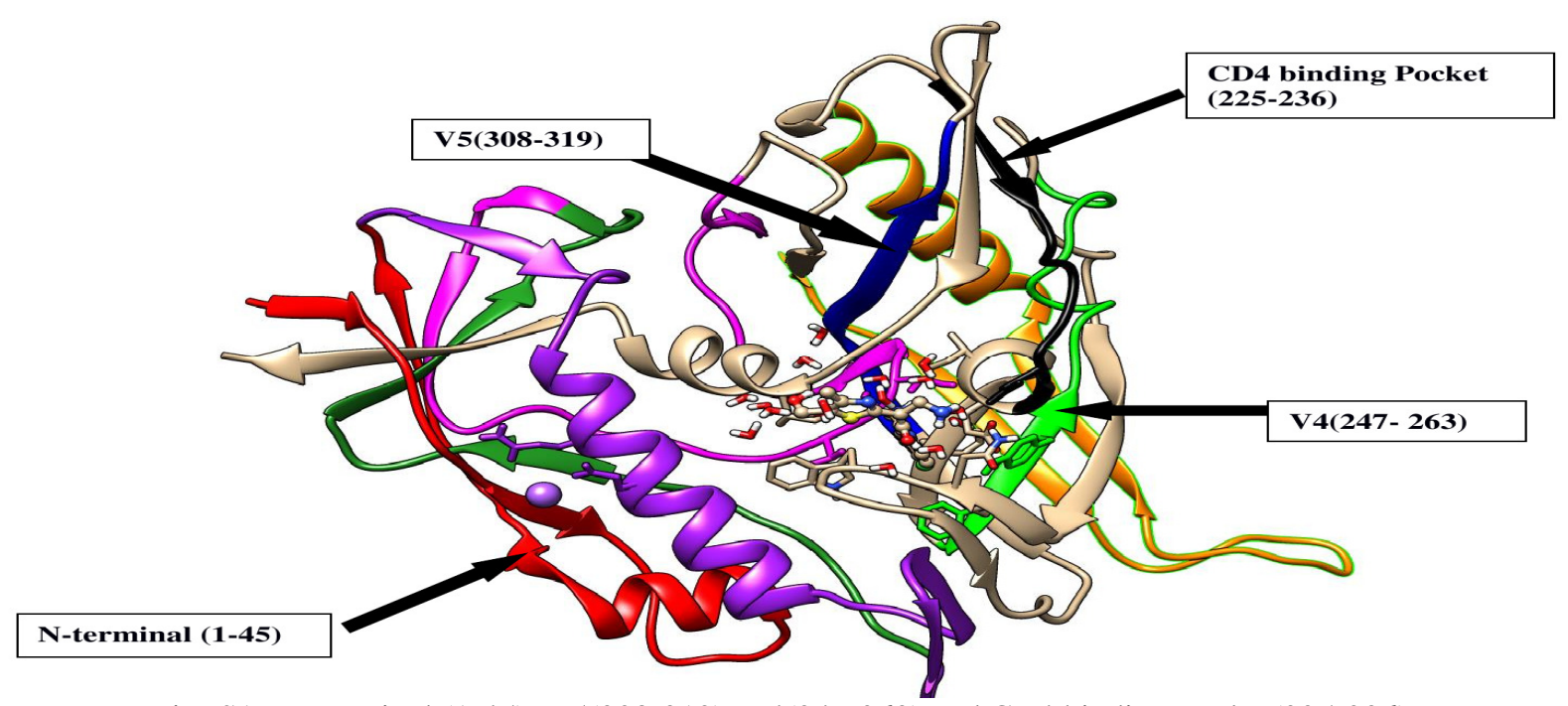

Fig.-S1: N-terminal (1-45), V5(308-319), V4(247-263) and CD4 binding Pocket(225-236)

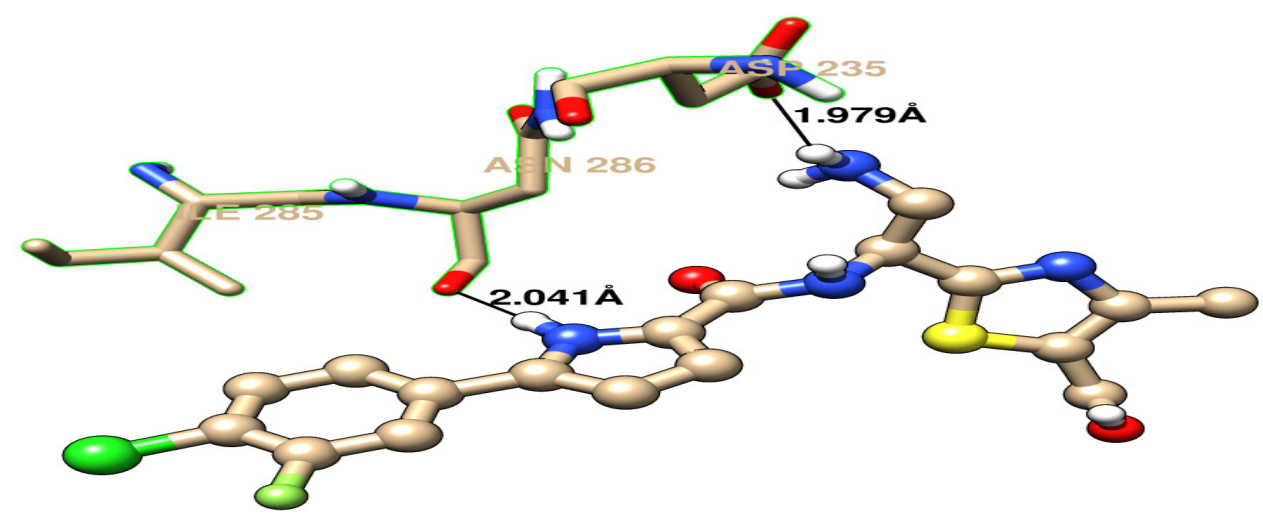

Fig.-S2: Hbond distance with surrounding residues.

\section{CONCLUSION}

The present study explores the effect of ligand 82M on gp120 protein with MD simulations and free energy calculations. By RMSD and RMSF we have studied that binding of $82 \mathrm{M}$ barely affects the $\mathrm{N}$ terminal which is the gp41 binding site, however, it restricts the flexibility of CD4 pocket. The thermo dynamical analysis using MMPBSA study shows that ligand $82 \mathrm{M}$ binding is mainly governed by hydrophobic and van der Waals interactions. The electrostatic interactions are relatively weak and it is only the Asn 286 which forms a strong H-bond with ligand 82M, and is mainly responsible for electrostatic interactions. In addition, Trp288 also provide a good energetic interaction via its hydrophobic side chain and is the main contributor to the increased van der Waals interactions. The residue-wise decomposition analysis finds few more amino acids such as: VAL139, THR141, GLU237, SER242, TYR251, ILE285, MET287, GLY290, GLY334, ASN335 and ILE336 as the key residues for the complex stabilization of the four ligands. These residues reside in the close vicinity of the binding site and therefore they are potentially important for the further drug discovery project as we can model a new drug which can interact more efficiently with them and maybe better binder than 82M. 
RASĀYAN J. Chem.

Vol. 11 | No. 1 | 405 - 412 | January - March | 2018

\section{REFERENCES}

1. F. Barre-Sinoussi, J.C. Chermann, F.Rey, M.T. Nugeyre,S.Chamaret, et al., Science., 220, 868 (1983)

2. R.C. Gallo, S.Z. Salahuddin, M. Popovic, G.M. Shearer, M. Kaplan, et al., Science., 224, 500(1984)

3. J. Liu, A. Bartesaghi, M.J. Borgnia, G. Sapiro, S. Subramaniam, Nature., 455, 109(2008), DOI: 10.1038/nature07159.

4. S.R. Wu, R. Loving, B. Lindqvist, H. Hebert, P.J. Koeck, et al., Proc. Natl. Acad. Sci., USA, 107, 18844(2010), DOI: 10.1128/JVI.00530-13

5. P. Sang, L. Yang, X. Ji, Y. Fu, S. Liu, PloS. One., 9(8), e104714(2014), DOI: 10.1371/journal.pone.0104714

6. A.L. Cunningham, H. Donaghy, A.N. Harman, M. Kim, S.G. Turville, Curr. Opin. Microbiol., 13, 524 (2010), DOI: 10.1016/j.mib.2010.06.002

7. P.M. Colman, M.C. Lawrence, Nat. Rev. Mol. Cell Biol., 4, 309(2003)

8. C.C. Huang, S.N. Lam, P. Acharya, M. Tang, S.H. Xiang, et al., Science., 317, 1930(2007)

9. C.C. Huang, M. Tang, M.Y. Zhang, S. Majeed, E. Montabana, et al., Science 310, 1025(2005), DOI: 10.1126/science. 1118398

10. P.D. Kwong, R. Wyatt, S. Majeed, J. Robinson, R.W. Sweet, et al., Structure., 8, 1329(2000)

11. P.D. Kwong, R. Wyatt, J. Robinson, R.W. Sweet, J. Sodroski, et al., Nature., 393, 648(1998), DOI: $10.1038 / 31405$

12. M. Pancera, S. Majeed, Y.E. Ban, L. Chen, C.C. Huang, et al., Proc. Natl. Acad. Sci., USA, 107, 1166 (2010), DOI: 10.1073/pnas.0911004107

13. F. Curreli, D.S. Belov, R.R. Ramesh, N. Patel, A. Altieri, A.V. Kurkin, A.K. Debnath, Bioorg. Med. Chem ., 24(22), 5988(2016)

14. F. Curreli, Y.D. Kwon, H. Zhang, D. Scacalossi, D.S. Belov, A.A. Tikhonov, I.A. Andreev, A. Altieri, A.V. Kurkin, P.D. Kwong, A.K. Debnath, J. Med. Chem., 58(17), 6909(2015), DOI: 10.1021/acs.jmedchem.5b00709

15. F. Curreli, Y.D. Kwon, D.S. Belov, R. Ramesh, A.V. Kurkin, A. Altieri, P.D. Kwong, A.K. Debnath , J. Med. Chem., 60(7), 3124(2017), DOI: 10.1021/acs.jmedchem.7b00179

16. D.A. Case, T.E. Cheatham, T. Daren, H. Gohlke, R. Luo, K.M. Merz, A. Onufriev, C. Simmerling, B. Wang, R.J. Woods, J. Comput. Chem., 26, 1668(2005)

17. C.I. Bayly, P. Cieplak, W. Cornell, P.A. Kollman, J. Phys. Chem., 97, 10269(1993)

18. W.D. Cornell, P. Cieplak, C.I. Bayly, P.A. Kollman, J. Am. Chem. Soc., 115, 9620(1993)

19. W.L. Jorgenson, J. Chandrashekhar, J.D. Madura, R.W. Imprey, M. Klein, J. Chem. Phys., 79, 926 (1983)

20. J.A. Izaguirre, D.P. Catarello, J.M. Wozanaik, R.D. Skeel, J. Chem. Phys., 114, 2090(2001)

21. H.J.C. Berendsen, J.P.M. Postama, W.F. van-Gunsteren, A. DiNola, J.R. Haak, J. Chem. Phys., 81, 3684 (1984)

22. J.P. Ryckaert, G. Cicotti, H.J.C. Barendsen, J. Comput. Phys., 23, 327(1977)

23. T. Darden, D. York, L. Pedersen, J. Chem. Phys., 98, 10089 (1993)

24. W. Humphrey, A. Dalke, K. Schulten, J. Mol. Graph. Model., 14, 33 (1996)

25. E.F. Pettersen, T.D. Goddard, C.C. Haung, G.S. Couch, D.M. Greenblatt, E.C. Meng, T.E. Ferrin, J. Comput. Chem., 25, 1605(2004), DOI: 10.1002/jcc.20084

26. V. Tsui, D.A. Case, Biopolymers., 56, 271(2001)

27. H. Gohlke, D. A. Case, J. Comput. Chem., 25, 238(2003)

28. F. Fogolari, A. Brigo, H. Molinari, Biophys. J., 85, 159(2003)

29. K.D. Dubey, A.K. Chaubey, R.P. Ojha, Biochim. Biophys. Acta., 1814, 1796(2011), DOI: 10.1016/j.bbapap.2011.09.012

30. K.D. Dubey, A.K. Chaubey, R.P. Ojha, Biochim. Biophys. Acta., 1834, 53(2013),DOI: 10.1016/j.bbapap.2012.08.014

31. K.D. Dubey, G. Tiwari, R.P. Ojha, J. Mol. Model., 23, 102(2017), DOI: 10.1007/s00894-017-3259-2

32. V. Pandey et al, Int. J. Recent Sci. Res., 9(1), 22913(2018), DOI: 10.24327/ijrsr.2018.0901.1360

[RJC-2050/2017] 